SITZUNGSBERICHTE DER SÄCHSISCHEN AKADEMIE DER WISSENSCHAFTEN ZU LEIPZIG Mathematisch-naturwissenschaftliche Klasse Band 115 . Heft 3

PAUL GÖRLICH

\begin{abstract}
DIE GESCHICHTLICHE ENTWICKLUNG DES WISSENSCHAFTLICHEN GERÄTEBAUS UND SEINE ZUKÜNFTIGE BEDEUTUNG
\end{abstract}

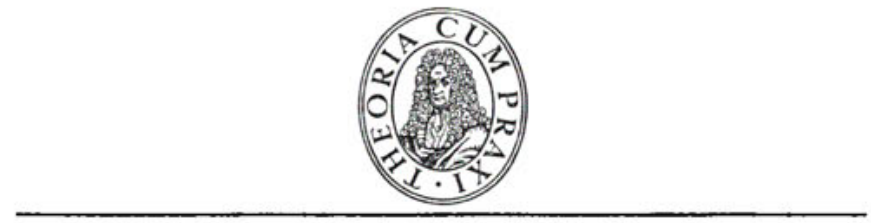

AKADEMIE-VERLAG - BERLIN 


\title{
SITZUNGSBERICHTE DER SÄCHSISCHEN AKADEMIE DER WISSENSCHAFTEN ZU LEIPZIG
}

\author{
MATHEMATISCH-NATURWISSENSCHAFTLICHE KLASSE
}

Band 109

Heft 1 Prof. Dr. ERICH RAMMLER, Uber die Theorien der Braunkohlenbrikettentstehung 1970. 38 Seiten - $13 \mathrm{Abb}$., davon 2 auf 2 Tafeln $-8^{\circ}-\mathrm{M}_{4}$,

Heft 2 Prof. Dr. Wolfgang Tutschke, Stammfunktionen komplexwertiger Funktionen 1970. 20 Seiten $-8^{\circ}-$ M $3, \hat{\imath}$

Heft 3 Dr. habil. GUNTher Eisenreich, Zur Syzygientheorie und Theorie des inversen Systems perfektt Ideale und Vektormodule in Polynomringen und Stellenringen 1970.88 Seiten $-8^{\circ}-M_{10, \varepsilon}$

Heft 4 Prof. Dr. med. RoLF EMMrich, Hochdruck und Hyperlipidämie (Hypercholesterinămie) als Risikc faktoren für die Entstehung der Arteriosklerosen

1971. 23 Seiten -10 Abbildungen -4 Tabellen $-8^{\circ}-\mathrm{M} 3, \sqrt{ }$

Heft 5 Prof. Dr. HANs Drischel, Biologische Rhythmen

1972. 57 Seiten -31 Abbildungen -1 Tabelle $-8^{\circ}-$ M 6,6

Heft 6 Prof. Dr.-Ing. Dr. h. c. KURT SCHWABE, Konzentrierte Elektrolytlösungen - Thermodynamisch und kinetische Eigenschaften 1972. 49 Seiten -27 Abbildungen -2 Tabellen $-8^{\circ}-M_{7,5}$

Heft 7 Prof. Dr. Wolfasa Tutschke, Konstruktion von globalen Lösungen mit vorgeschriebenen Singu larităten bei partiellen komplexen Differentialgleichungen 1972. 24 Seiten $-8^{\circ}-M_{4,5}$

\section{Band 110}

Heft 1 Prof. Dr. h. c. PAUL Görlich, tber die Laser und ihre Anwendung

1972. 24 Seiten $-8^{\circ}-\mathrm{M} 2,31$

Heft 2 Prof. Dr. Hasso Essbach, Zum Problem der Tumoren im Kindesalter

1972. 24 Seiten -11 Abbildungen auf 10 Kunstdrucktafeln $-8^{\circ}-$ M 6, -

Heft 3 Prof. Dr. med. WAlter Brednow, Zur Anthropologie des Schwindels

1973. 17 Seiten -2 Abbildungen auf 2 Kunstdrucktafeln $-8^{\circ}-$ M 2,5c

Heft 4 Prof. Dr. h. c. PAUL GöRlich, Betrachtungen über den Wissenschaftlichen Gerätebau

1972. 39 Seiten $-8^{\circ}-$ M 3,-

Heft 5 Prof. Dr. ЕкICH RAMMLER, Einige Betrachtungen über Erdgas

1974. 43 Seiten -8 Abbildungen -3 Tabellen $-8^{\circ}-$ M 4,50

Heft 6 Prof. Dr. Gustav E. R. SchuLze, Zur Rolle des Einfachheitsprinzips im physikalischen Weltbild

1974. 23 Seiten -4 Abbildungen $-8^{\circ}-\mathbf{M} 2,50$

Heft 7 Prof. Dr. med Rolf Emaricl, Zwischen Leben und Tod. Arztliche Probleme der Thanatologie 1974. 22 Seiten -2 Abbildungen -4 Tabellen $-8^{\circ}-\mathbf{M} 3,5 \mathrm{C}$

Band 111

Heft 1 Prof. Dr. WLHELM MaIeR, Vom Erbe Bernhard Riemanns

1975. 16 Seiten $-8^{\circ}-$ M $2,5 \mathrm{C}$

Heft 2 Prof. Dr. med. HANs DrIsohrL, Organismus und geophysikalische Umwelt

1975. 50 Seiten -25 Abbildungen -1 Tabelle $-8^{\circ}-$ M 7,-

Heft 3 Prof. Dr. Maria Hassy, Zum Begriff des allgemeinen Produkts von Kategorien 